\title{
Wireless Sensor Networks: A Comprehensive Comparison of Routing Protocols and Energy Efficient Techniques
}

\author{
Musharaf Chaudhary \\ Bachelor of Electrical Engineering \\ Bahria University, \\ Islamabad, Pakistan
}

\begin{abstract}
Wireless sensor network is a network of randomly distributed autonomous sensors to oversee different environmental conditions. Contemporary enhancements in the field of wireless communications, memory, processor and radio technology have enabled the growth of low-cost sensor networks and sensor nodes which is proficient in sensing, communication and calculation. These sensor networks have tremendous applications in the various fields of life (Medical, military, industry, surveillance systems, etc.). Because of this large number of applications, there are copious routing protocols and techniques under which these sensor networks are accomplished. This survey presents the current state of the art of WSN's, a collective knowledge of those routing protocols (S-MAC, MAC, IEEE 802.11, LEACH, and SPIN) and energy efficient techniques (clustering technique, directed diffusion technique) which are being implemented for their competent functioning. Further, those routing protocols will be discussed briefly and their different aspects and characteristics will be compared. Finally, a better idea among all these routing protocols and energy efficient techniques for WSN's will be presented.
\end{abstract}

\section{Keywords}

Sensor nodes, routing protocols, wireless sensor networks, clustering algorithm and medium access control.

\section{INTRODUCTION}

Wireless Sensor Network (WSN) is a very in-demand research topic for researchers. In recent years, the field of wireless communications is becoming faster and drawing nearer to the extreme height of climax. Advancements in the field of processor, memory, wireless, radio technology and semiconductor fabrication technology has enabled electronic devices to be smaller and cheaper. The innovation of consuming less power for the better functioning allows the development of the mini devices known as "Sensor Nodes". These sensor nodes use wireless technology for their operation, hence they are also referred to as "Wireless Sensor Nodes". And the cluster or a mesh type network of all the nodes interconnected with each other in a specific area is known as "Wireless Sensor Network". This wireless mesh type network is composed of hundreds and thousands of compact sensing devices called sensor nodes.

A Sensor node is a mini compact device which has its own memory, processor, antenna, sensors, battery and radio or $\mathrm{Wi}-\mathrm{Fi}$ device. These nodes have the ability to wirelessly communicate with its server and also with other nodes in a specific area. It has its own computing power to calculate the temperature, humidity and other parameters as well. A long time battery which acts as a source of power and helps it working continuously for more than two or three years which mostly depends upon the device use age. A small and cheap device having all the abilities in it, this quality differentiates the sensor nodes from all other monitoring devices. Figure 1 shows a typical block diagram of a sensor node.

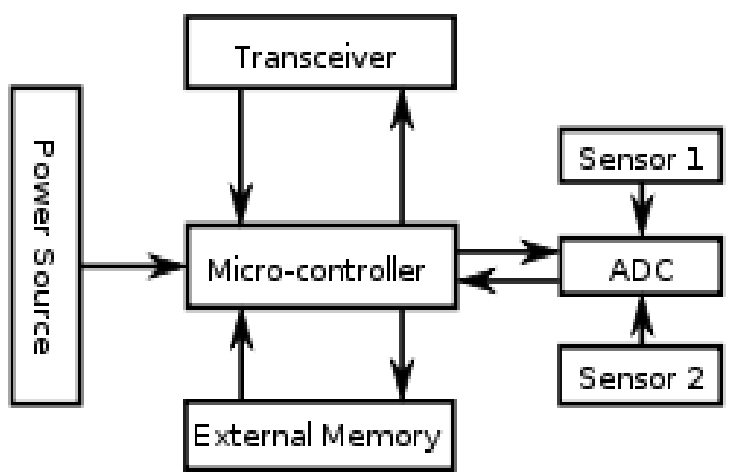

Figure 1. Block Diagram of a Sensor Node

WSN's have enormous applications and implementations in almost all the fields of life, such as in surveillance systems, military purposes [5], medical, household, and also in industrial monitoring. Due to the great number of applications, everyone requires these sensor networks to be very efficient in working, more reliable, less energy consuming and long lasting. There are various routing protocols and different energy efficient techniques such as Medium Access Control Protocol (MAC) [2], which were implemented to get a beneficial output. Here we discuss about a collaborative knowledge of all those routing protocols and energy efficient techniques which are being implemented for the efficient functioning of the WSN's.

Previously, a lot of work has been done on wireless sensor nodes and WSN's, their routing techniques and protocols. Different researchers have discussed the problem and propose the solution in their own way. Wei, and others presented a novel technique and routing protocol, S-MAC protocol, which is more advanced and improved version of MAC protocol [8]. In this protocol, they have introduced a new energy efficient technique for WSN's and mainly concentrated on energy conservation and self-configuration, per node fairness is their primary goal. Similarly, many other researchers had spent their time in discovering the algorithms like clustering algorithm, directed diffusion. 
This paper contributes towards WSN's by collectively and briefly explaining all those routing protocols, algorithms and energy efficient techniques which were implemented for their competent functioning. Also by listing and mentioning all those protocols and techniques, then briefly explaining their attributes and aspects. Finally, in the light of all the discussions, a better idea has been proposed.

\section{RELATED WORKS}

WSN's are attracting the researchers due to their monstrous applications in almost every field of life like surveillance systems, medical, military purposes, industrial control monitoring and tracking purposes. Sensing and wireless qualities of the sensor nodes make them more fascinating and attractive. In parallel, there are lots of routing protocols and energy efficient techniques for their efficient functioning. Previously, different researchers have discussed about the various routing protocols like MAC protocol [2], IEEE 802.11 and S-MAC protocols, and also work on energy efficient techniques such as Clustering and Directed Diffusion techniques [3] to make the WSN's more productive and economical. Some people also gave a brief description of the EAR (Eaves-drop-And-Register) algorithm. In the light of the above scenarios, concluding to one point that due to the sensing and data routing technology, WSN's are becoming the crucial need of the modern era.

\section{WIRELESS SENSOR NETWORKS}

\subsection{Communication Architecture}

The sensor nodes are normally distributed in a sensor field such that it looks like they are scattered everywhere. Each of these sensor nodes has the capability to collect and route data back to the sink. Data is routed back to the user by a multi-hop infrastructure architectures, like mesh networks, to the sink [1]. The sink, then communicates with the task manager node via the Internet or satellite. The design of the sensor networks is affected by many factors, including scalability, operating environment, production costs, sensor network topology, transmission media, fault tolerance, hardware constraints and power consumption. The communication architecture of a WSN's is explained in the figure 2 [1].

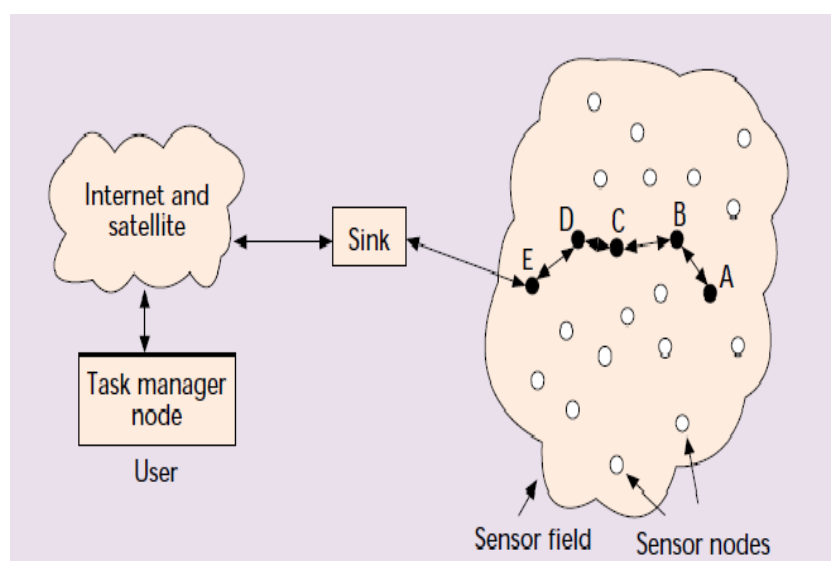

Figure 2. Communication Architecture of WSN

\subsection{Sensor Node Function Architecture}

Figure.3 shows the generic function architecture of a sensor node. It mainly consists of four sections or portions, namely Sensing portion, computing, communication and power section. Sensory section consists of a sensor unit. It has various types of actuators and sensors to sense conditions and parameters like temperature, humidity, motion sensors etc. It may also include small monitoring cameras to record a video of any happening. Computing section consists of processor, memory, RAM, operating protocol layers and converters. The main function of this section is to process the information received from the sensory section, convert it into useful format and then send it to the communication portion to route it on the server [4].

The foremost purpose of communication section is to connect the sensor nodes with its server and overall wireless network through wireless or radio and route all the useful data. It mainly consists of an antenna, wireless or radio transceiver. Power section has a vital role in all the given scenario in such a way that it provides the power to each section to operate and perform its functions. It mainly consists of a battery which is capable of providing a continuous power supply to the node for a long time. This a generic architecture on which all the sensor nodes are operating [1].

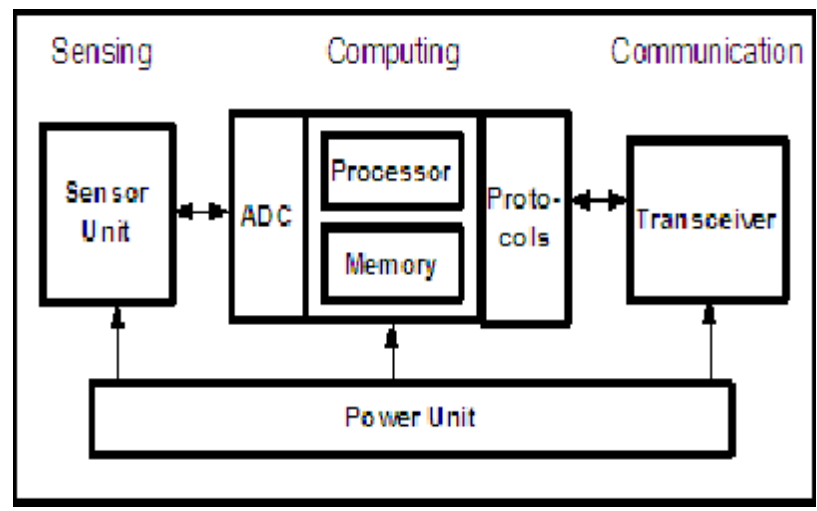

Figure 3. Sensor Node Architecture

\subsection{Classification of Routing Protocols}

There are different routing protocols and energy efficient techniques which are being implemented for the beneficent working of WSN's [9]. These protocols are further divided into different classes and sections depending upon their characteristics and properties. Figure 4 and 5 briefly described them [9]. 


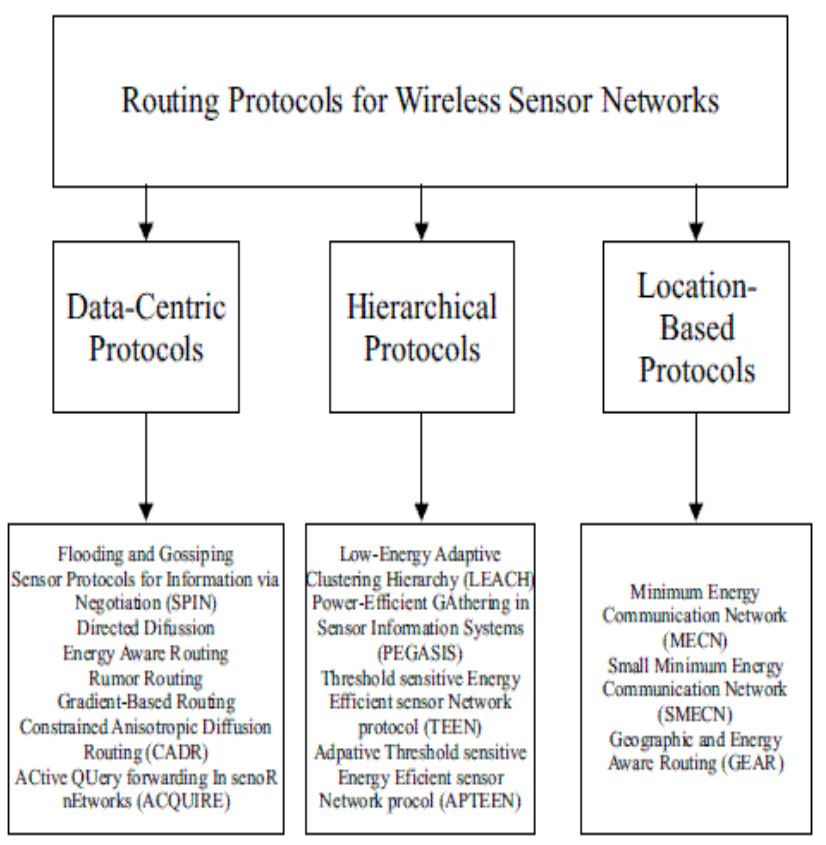

Figure 4. Routing protocols

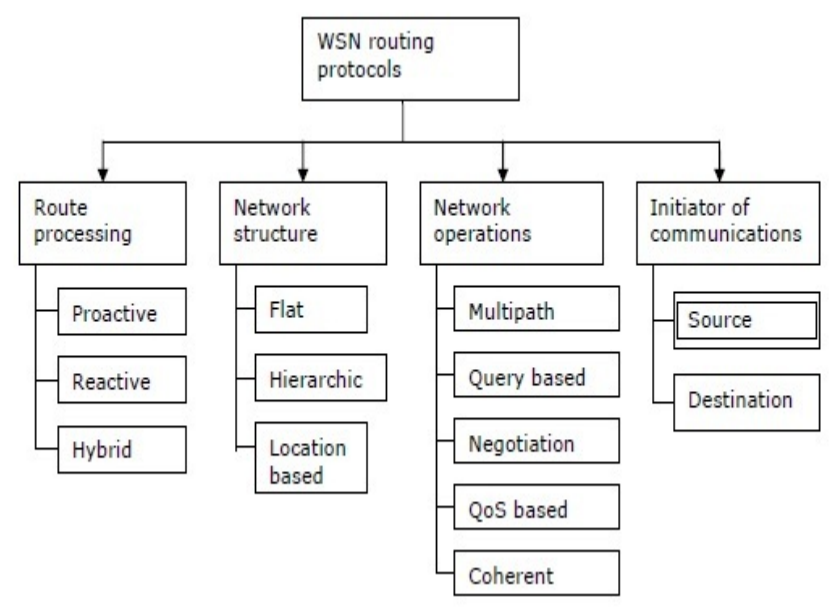

Figure 5. WSN's Protocols

\section{ENERGY-EFFICIENT ROUTING PROTOCOLS}

Due to the immense applications of WSN's in the all fields of life, its demand is increasing day by day rapidly. For this purpose, scientists and researchers are working to improve them as well as developing different efficient techniques for the economic functioning of wireless nodes. All those protocols and algorithms which are being implemented are briefly discussed here.

\subsection{IEEE 802.11}

IEEE 802.11 is a set of MAC and physical layer (PHY) specifications for implementing WLAN computer network in 2.4, 3.6, 5 and $60 \mathrm{GHz}$ frequency bands. It is a most common, most popular and most frequently used routing protocol in data communications and computer networking. It gives $2 \mathrm{Mbps}$ transmission speed and uses either frequency hopping spread spectrum (FHSS) or direct sequence spread spectrum (DSSS). WSN's are also being implemented under this protocol, WLAN built for sensor nodes for any specific area under observation uses this protocol layer to transmit the data across its servers [6]. A typical IEEE 802.11 internet routing protocol is shown in the figure 6 , in which different user clients are connected to one LAN through the base station.

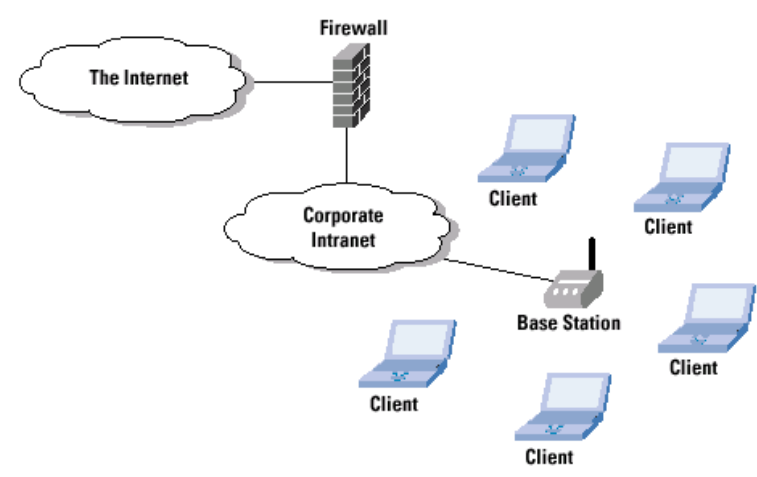

Figure 6. IEEE 802.11 Architecture

Table 1 shows the normal physical standards which are being implemented for IEEE 802.11.

Table 1 Physical Standards of IEEE 802.11

\begin{tabular}{|c|l|c|c|c|c|c|}
\hline Release date & Standard & Band (GHz) & Bandwidth (MHz) & Modulation & $\begin{array}{c}\text { Advanced antenna } \\
\text { technologies }\end{array}$ & $\begin{array}{c}\text { Maximum } \\
\text { date rate }\end{array}$ \\
\hline 1997 & 802.11 & 2.4 & 20 & DSSS, FHSS & N/A & $2 \mathrm{Mbits} / \mathrm{s}$ \\
\hline 1999 & $802.11 \mathrm{~b}$ & 2.4 & 20 & DSSS & N/A & $11 \mathrm{Mbits} / \mathrm{s}$ \\
\hline 1999 & $802.11 \mathrm{a}$ & 5 & 20 & OFDM & N/A & $54 \mathrm{Mbits} / \mathrm{s}$ \\
\hline 2003 & $802.11 \mathrm{~g}$ & 2.4 & 20 & HSSS, & OFDM & $54 \mathrm{Mbits} / \mathrm{s}$ \\
\hline 2009 & $802.11 \mathrm{n}$ & $2.4,5$ & 20,40 & OFDM & $\begin{array}{c}\text { MIMO, up to four } \\
\text { spatial streams }\end{array}$ & $600 \mathrm{Mbits} / \mathrm{s}$ \\
\hline 2012 & $802.11 \mathrm{od}$ & 60 & 20,60 & SC, OFDM & Beamforming & $6.76 \mathrm{Gbits} / \mathrm{s}$ \\
\hline 2013 & $802.11 \mathrm{oc}$ & 5 & $40,80,160$ & OFDM & $\begin{array}{c}\text { MIMO, MU-MIMO, } \\
\text { up to eight spatial } \\
\text { streams }\end{array}$ & $6.93 \mathrm{Gbits} / \mathrm{s}$ \\
\hline
\end{tabular}




\subsection{MAC Protocol}

Medium Access Control (MAC) [2] is an energy-efficient technique. It is one of the two sub-layers that make up Data Link layer of the OSI model of computer networking. The MAC layer is responsible for routing and receiving data to and from the one Network Interface Card (NIC) to another channel connected. MAC sub-layer uses the MAC protocols to ensure the delivery of message sent from different nodes across one network should not collide with each other. OSI seven layer model is shown in the figure 7 [2]. It has different layers, each having its own specific function, all of them perform its own task in communication and data routing.

\section{The Seven Layers of OSI

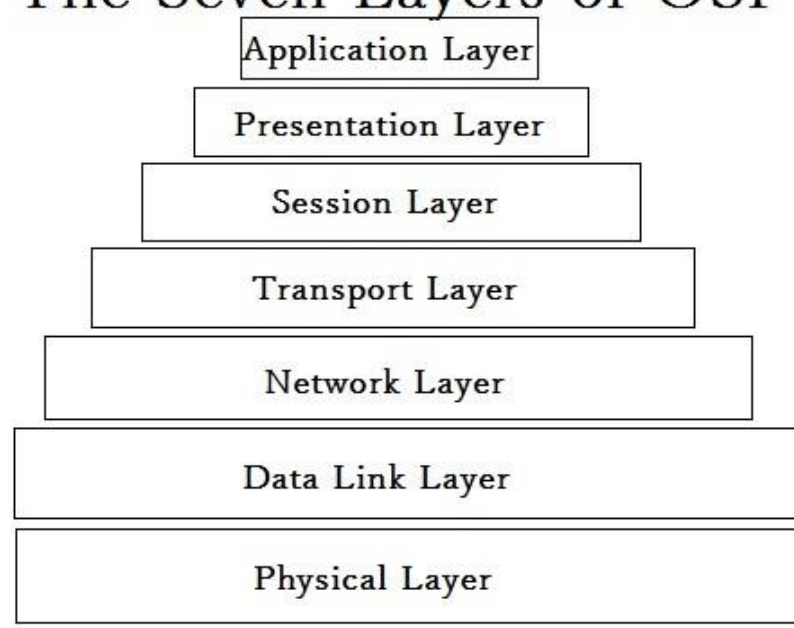

Figure 7. OSI Seven layer Model

\subsection{S-MAC Protocol}

Sensor-MAC protocol is also an energy efficient protocol for WSN's. It is explicitly designed for WSN's where energy conservation is the primary goal. In this protocol, good scalability and collision avoidance are the two main abilities where per node fairness and latency are less important. It produces good scalability and reduces collisions by combining scheduling and contention schemes [8]. In this protocol, sensor nodes are deployed in an ad hoc fashion and they remain inactive for longer times to save energy, but whenever something is detected it suddenly got active, sense the happening, route the useful information and again goes to sleep mode. In this way, we are able to save maximum energy. SMAC messaging and routing scenario is explained in the figure 8 [8].

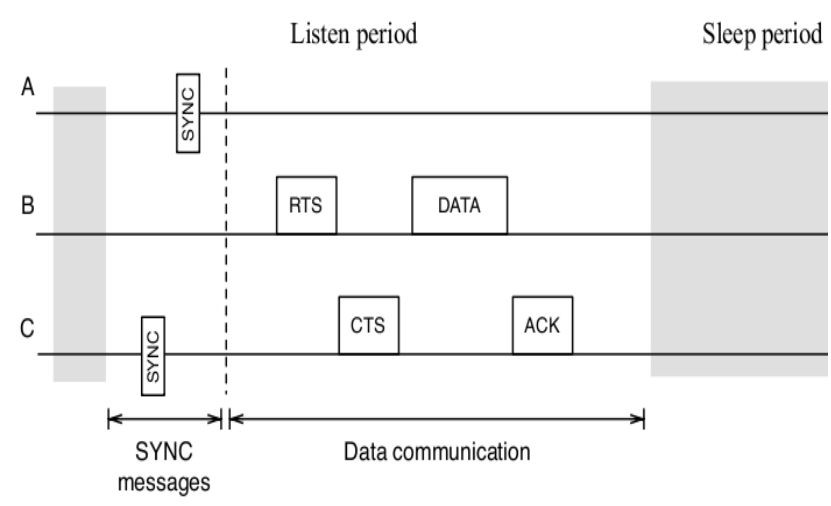

Figure 8. S-MAC messaging scenario

\subsection{LEACH}

LEACH (Low Energy Adaptive Clustering Hierarchy) is a technique which is specially designed for sensor networks in which the user can be able to remotely monitor the under observation area. In this situation, the useful data must be sent from the sensor node to the central base station and then the user access that data from that station. It is often located far from the sensor nodes and close to the end-user. In this protocol, there are a lot of benefits and plus points which the user can achieve like more than 1000 nodes can be used in a network, system lifetime is increased to a greater extent, network coverage is maximized, and uniform battery operated nodes are used. Figure 9 shows a communication architecture of the LEACH protocol in which all the individual cluster nodes are connected to a cluster head and that cluster head is connected to a nearby base station. End-user can access and monitor all the activity by connecting to that base station.

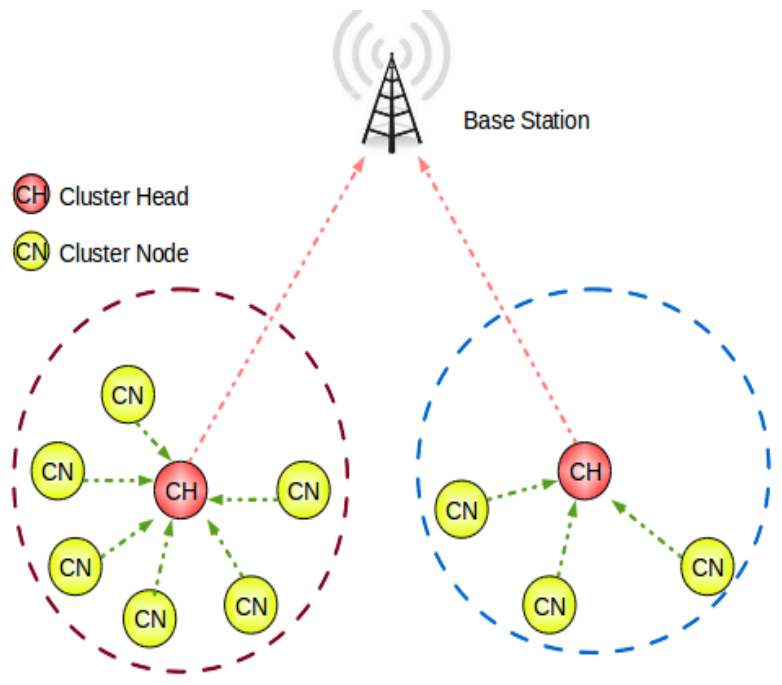

Figure 9. LEACH protocol communication architecture

\subsection{SPIN}

SPIN (Sensor Protocols for Information via Negotiation) is a class of routing protocols for WSN's in which the data is efficiently routed to the wireless network without any collision, damage, and loss. Conventional data approaches like flooding and gossiping, wastes valuable information and energy reserves as well, sending redundant information throughout the network.

\subsection{Clustering Technique}

Clustering is an energy efficient routing technique used in WSN's. In this technique, all the individual sensor nodes in a specific area under observation are arranged in the form of groups, called as clusters, so that the sensors only communicate information to cluster heads, and the cluster heads, then communicate and forward the aggregated information to its nearest server, sink or processing center. In this way energy is saved to a much greater extent and the chances of loss of data are minimized [7]. Figure.10 shows the clustering algorithm technique. 


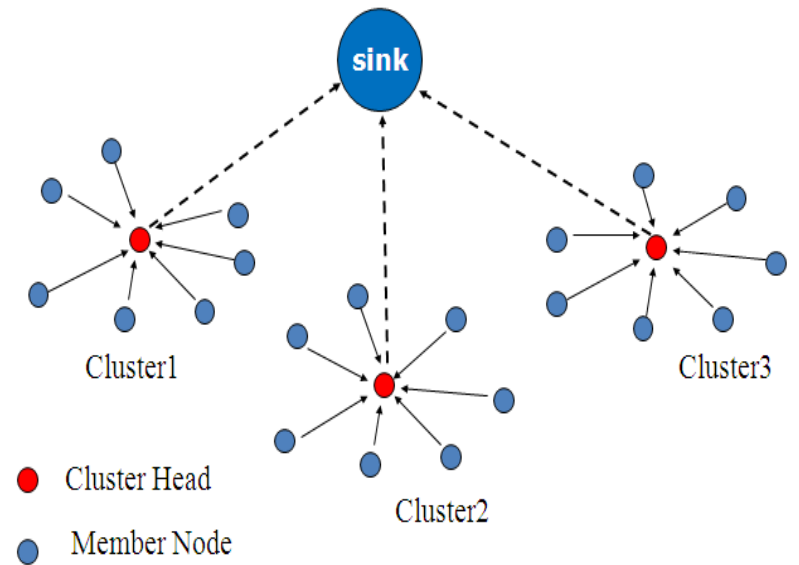

Figure 10. Clustering Technique

\subsection{Directed Diffusion Technique}

Directed diffusion is a novel data centric, data dissemination paradigm for WSN's [3]. Directed diffusion has some novel features, named as data centric, data dissemination, reinforcement-based adaptation to the empirically best path, and in-network data aggregation and caching. These features can make them highly energy-efficient and enable robust dissemination in dynamic sensor networks, while at the same time minimizing per node configuration that is a characteristic of today's network.

Directed diffusion is also referred to as the scalable and robust communication paradigm for WSN's. It has several different elements, data naming, an interest for naming data is made through sensing tasks, and then the gradients are set up within the wireless network which is designed specially to draw events. Below figure 11 explains the diffusion technique for WSN's in which a source node is deployed in an event and other nodes are present outside that event and are interconnected with each other. Data from the source node is routed to the sink thorough this directed diffusion technique.

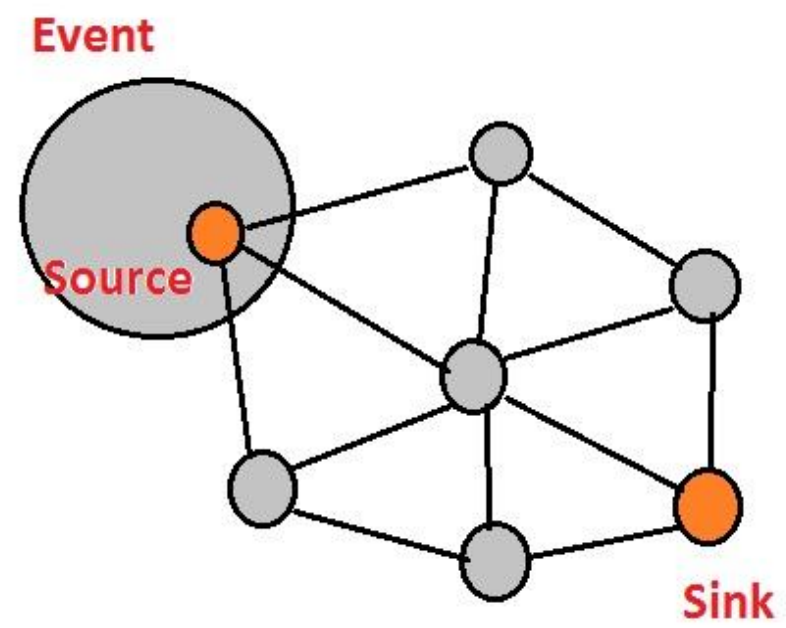

Figure 11. Directed diffusion

\section{RESULTS}

WSN's have enormous applications in the modern era which leads them to a very high level of advancement. A small mini device having a technology which is used by every type of department. In the military, it is used for surveillance systems, monitoring and controlling any targeted area, in industry, it is used for controlling and remotely monitoring all the activity. Similarly, in the medical department, it is used to monitor the activity happening in the patient ward.

The results have been shown here, which have drawn from all of the above discussions on the routing protocols and energy efficient techniques.

\subsection{IEEE 802.11}

In figure 12, a graph has been plotted between the energy consumption in joules and wait time in milliseconds. The IEEE 802.11 wireless LAN access standard offers a power saving mode of operation in which both the wireless station and the access point cooperate to conserve energy. The wireless station informs the access point closest to it to switch to power saving mode, and the wireless station switches the Wireless Network Interface Cards (WNIC's) to low power sleep mode, hence conserving the energy.

This energy conservation depends upon the Wait time intervals (the time between the transmission of poll message and receipt of data packet). As the wait time interval is long, energy conservation is affected. If the wait time is less than $100 \mathrm{~ms}$, energy consumption is less which is around 40-60 joules, but when this time increases from $100 \mathrm{~ms}$ energy consumption increases to 100-140 joules. This graph shows that wait drastically affects the energy conservation. However, larger wait times do not offer energy conservation.

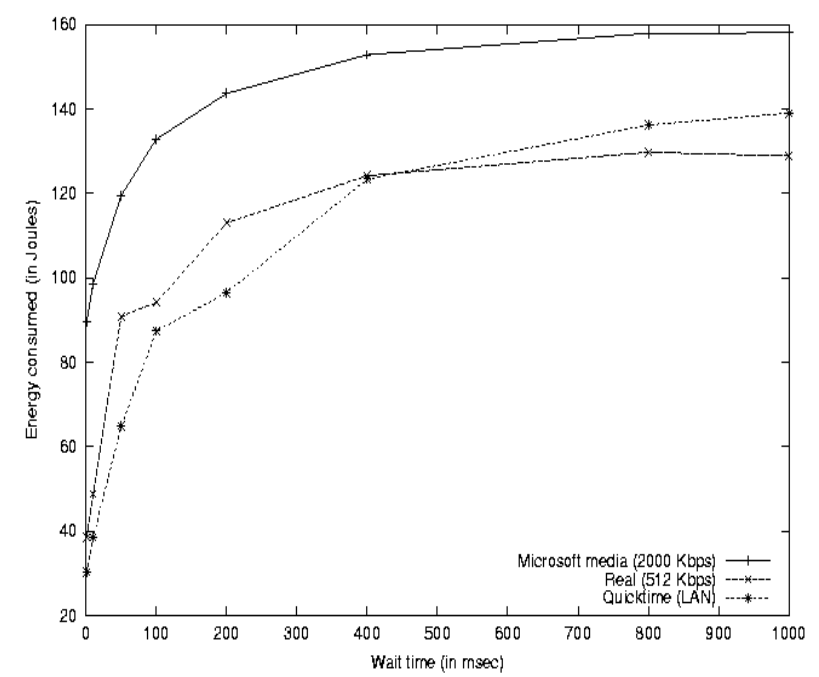

Figure 12. Energy consumption graph for IEEE 802.11

\subsection{MAC}

This section briefly discusses how MAC protocol is inefficient in saving the energy and how it reduces its consumption in wireless sensor nodes. For this purpose, in the first graph, the number of node count is plotted against energy consumption and in the second graph, node count is plotted against the time for different algorithms of the MAC layer.

In the first graph, it is clear that the node count is directly proportional to the amount of energy consumed in mill joules. As the number of nodes count increases, energy consumption increases proportionally and a straight line is obtained. It is shown in figure 13 (a). 


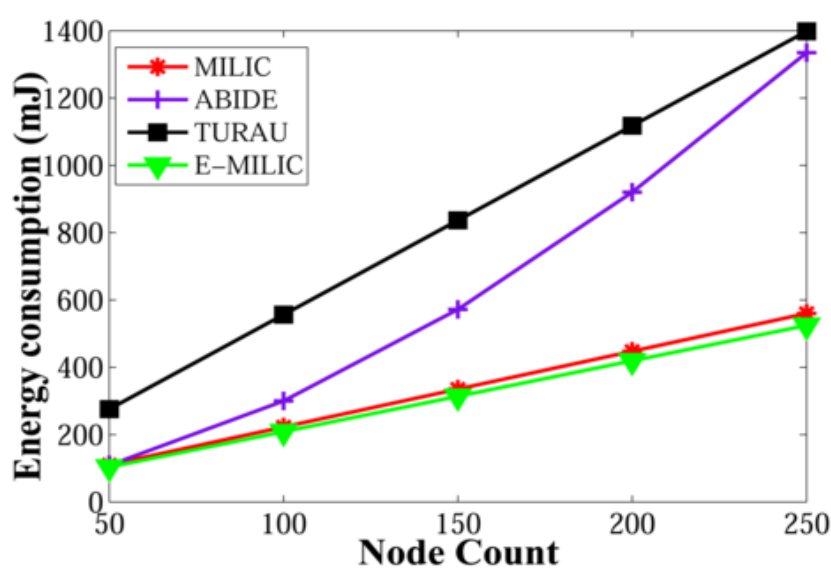

Figure 13. (a) Energy consumption graph for MAC protocol

In this graph, the number of node count is plotted against delay time in seconds. As the number of node count is increased, the delay time between transmission and receiving of data is increased and in this way more energy is consumed. This is explained through figure 13 (b).

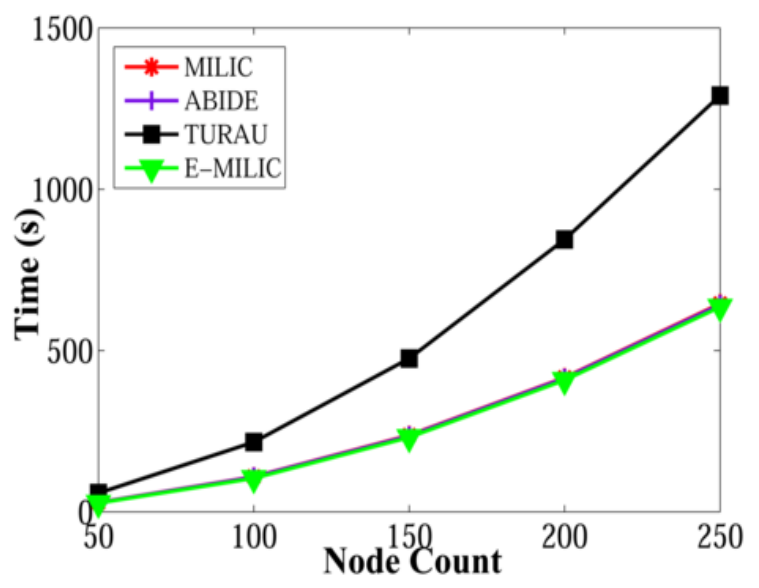

Figure 13. (b) Node count verses time

\subsection{S-MAC}

While discussing the results obtained from the S-MAC protocol, consider plotting a graph of those parameters which are making it different from all others. Here the throughput in Bits/s and inter-packet time required in seconds for S-MAC CSMA backoffs has been shown. It is clear from the graph that the throughput is maximum when the inter-packet delay time is less and as the time is increased throughput is decreased gradually. It is explained in figure 14.

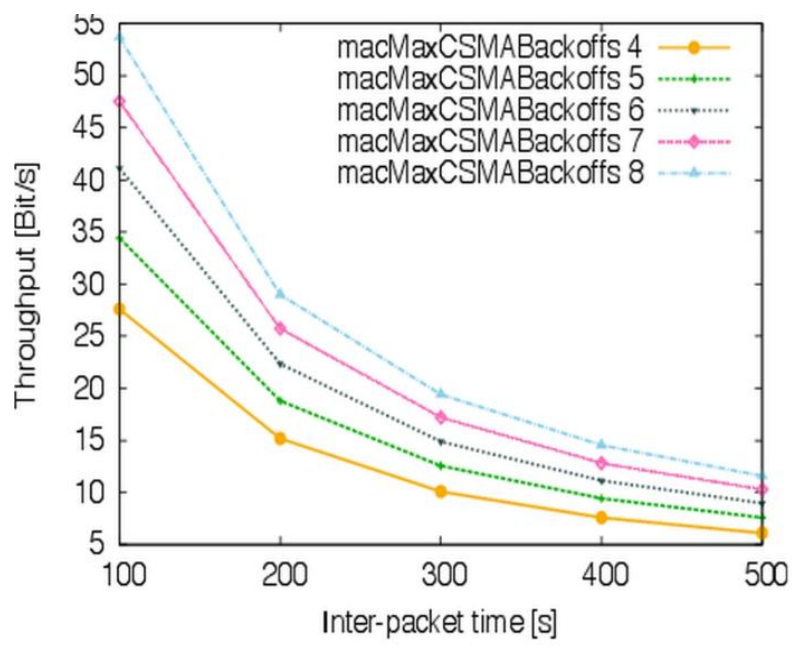

Figure 14. S-MAC throughput graph w.r.t time

\subsection{LEACH}

Below figure (15) shows the comparison of the performance of the LEACH protocol with other protocols in terms of energy conservations. It is clear from the graph that by using gateway nodes for the transmission of data from cluster heads to the sink, energy is decreased gradually. This is due to the gain of the energy dissipated by the cluster heads to the base station. It is obvious from the graph that LEACH protocol is not much efficient routing protocol in terms of energy savings.

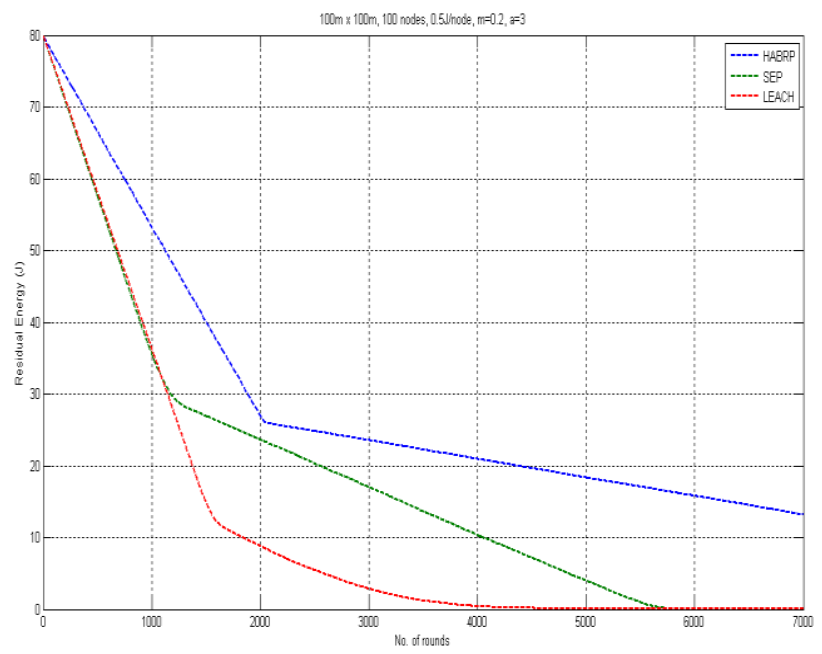

Figure 15. LEACH protocol

\subsection{Clustering Technique}

In energy-efficient clustering technique, using MATLAB, results were plotted on three different graphs which shows that how it conserves the energy. It is obvious that as the number of nodes in any specific area or in a given wireless network is increased, the average distance between the nodes is decreased. This is due to the fact that all the nodes come closer to each other due to the dense environment and in this way energy is conserved. In the last graph, it is clear that in a cubic shape cluster all the sensor nodes are arranged in such a way that their cluster is much more energy efficient than any other cluster. The whole phenomenon is explained in figure 16 (a) and 16 (b). 

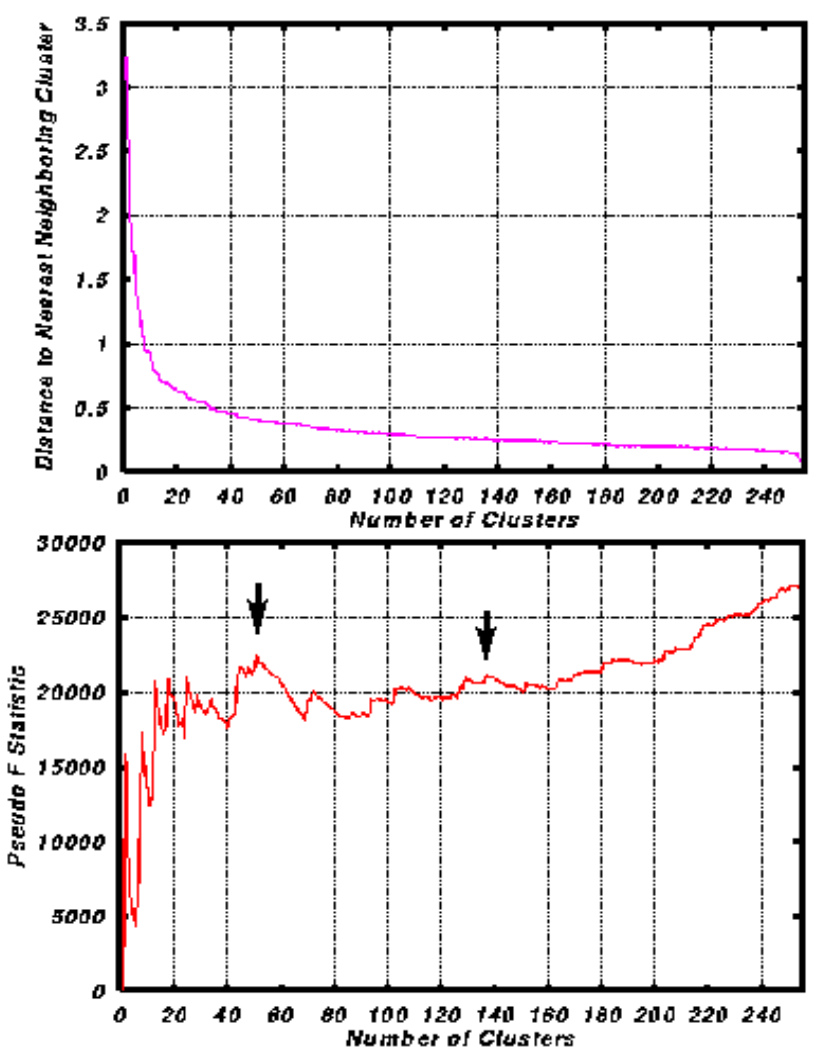

Figure 16 (a). Clustering technique energy conservation graph

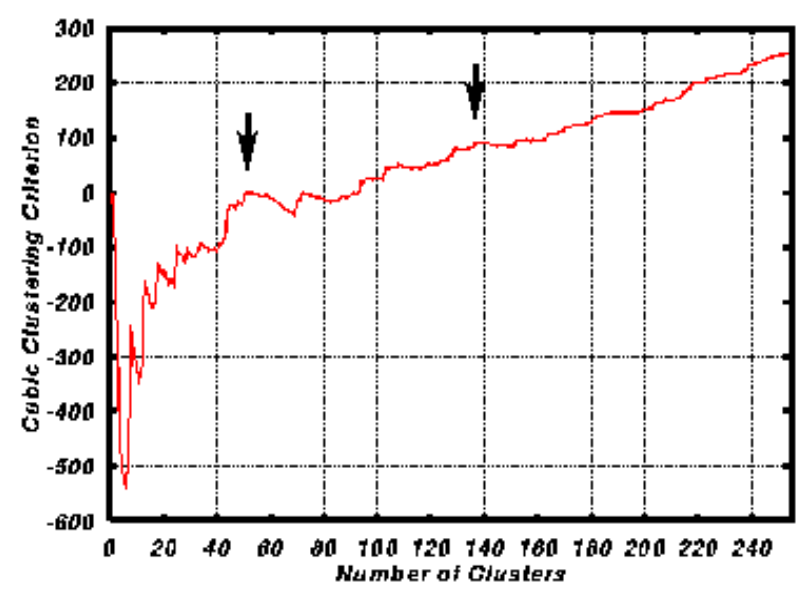

Figure 16 (b). Clustering technique energy conservation graph

\subsection{Directed Diffusion}

In a directed diffusion technique, results has been plotted on a graph of the number of nodes against the percentage of remaining energy of a wireless network. It is clear that as the number of nodes is less in any directed diffusion network, its energy consumption is less and conserved for a long time, but if the number of nodes is increased, not only the consumption increases but also it is conserved for a little interval of time. It is explained in figure 17.

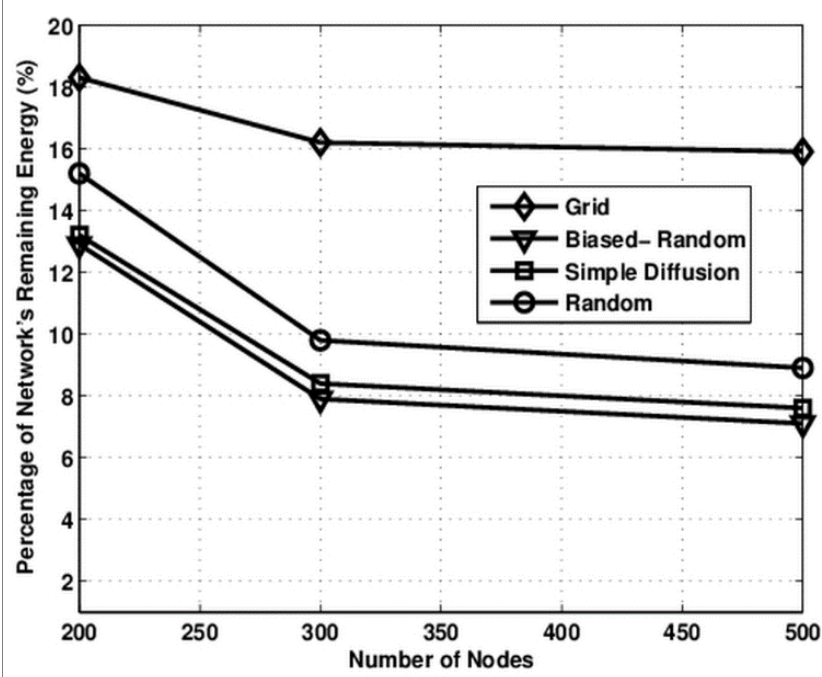

Figure 17. Graph of Directed Diffusion network energy

\subsection{Hop Distribution}

Figure 18 shows a plot between hop distribution phenomenon for Shortest Path (SP) and Minimum Transmission (MT) protocols. Firstly, the hop counts were calculated for sensor nodes and then plotted them against the number of sensor nodes involved and their combined hop count.

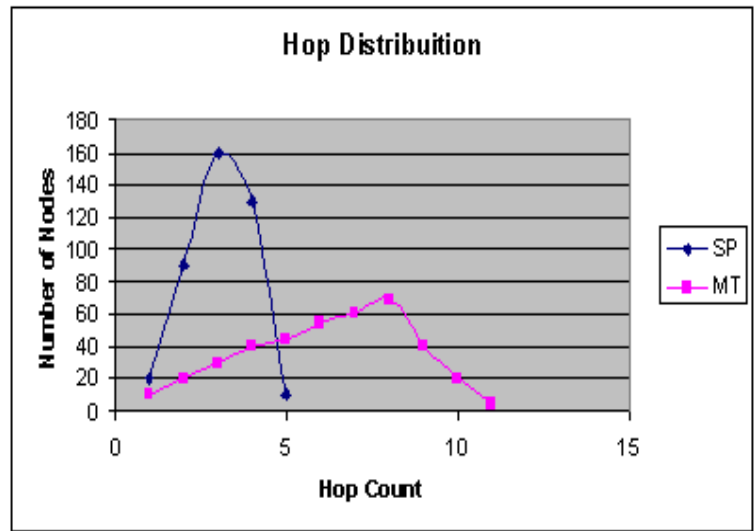

Figure 18. Hop Distribution

In figure 19, graphically represented the outputs of four channels and the battery lifetime when the number of samples per waveform is increased. It is obvious from the graph that when the number of samples per waveform are less for all the channels, life time of the battery is very large and it works for a longer duration of around 2 or 3 years. But as the number of samples per waveform is increased duration of the battery is decreased. 


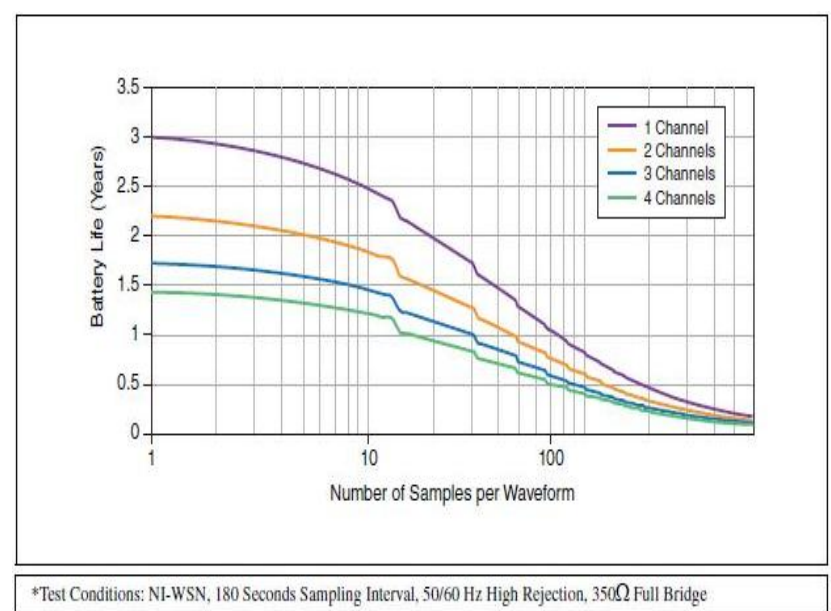

Figure 19. Battery consumption as per sample

In the light of all the discussions made regarding energy efficient techniques and routing protocols for WSN's, S-MAC protocol is the best protocol among all. It senses the current situation, activates the sensor from sleep mode to operation mode, process the information and route it to the sink or nearby station and again goes to sleep mode to save energy. In this way maximum energy is conserved and more efficiently the work is done.

\section{PERFORMANCE ANALYSIS}

While analyzing the performance of results, we should consider some basic properties and characteristics of the routing protocols and energy-efficient techniques. All those properties are listed in Table 2. Firstly, the names of protocols which are considered. Secondly, their type of communication either it is TDMA (Time Division Multiplexing), CDMA (Code Division Multiplexing), or CSMA (Carrier Sense Multiple Access). Then the energy conservation and energy awareness characteristics either it holds for this protocol or not were discussed. Then communication speed which is attained by applying this routing protocol, it is the overall communication speed which includes data transmission as well as receiving speed.

Traffic Adaptivity is also a main characteristic of a good routing protocol that what will it do when a traffic load comes in the way, whether it is adaptive to traffic handling or not. Then, synchronization of the nodes connected in the network while using this protocol, either it is local, network wide or not synchronized with any other server or node. Afterwards, complexity of the network and the protocol which is being implemented. Finally, the scalability of the routing protocol, either it is good, weak or bad is listed in the table below.

Table 2 Routing Protocols in WSN's

\begin{tabular}{|c|c|c|c|c|c|c|c|}
\hline Protocol Name & Type & $\begin{array}{c}\text { Energy } \\
\text { Awareness }\end{array}$ & $\begin{array}{c}\text { Communication } \\
\text { Speed }\end{array}$ & $\begin{array}{c}\text { Traffic } \\
\text { Adaptivity }\end{array}$ & Synch. & Complexity & Scalability \\
\hline S-MAC & $\begin{array}{c}\text { TDMA/ } \\
\text { CSMA }\end{array}$ & Yes & $200 \mathrm{Mbps}$ & Yes & Locally & High & Good \\
\hline MAC & CSMA & Yes & $150 \mathrm{Mbps}$ & Yes & Locally & High & Good \\
\hline IEEE 802.11 & CSMA & No & $300 \mathrm{Mbps}$ & No & $\begin{array}{c}\text { Network } \\
\text { Wide }\end{array}$ & Moderate & Weak \\
\hline LEACH & TDMA & Yes & $100 \mathrm{Mbps}$ & No & Locally & Moderate & Weak \\
\hline SPIN & CSMA & No & $100 \mathrm{Mbps}$ & No & None & Low & Weak \\
\hline Clustering & $\begin{array}{l}\text { CDMA/ } \\
\text { TDMA }\end{array}$ & Yes & $50 \mathrm{Mps}$ & Yes & $\begin{array}{c}\text { Network } \\
\text { Wide }\end{array}$ & Low & Good \\
\hline $\begin{array}{c}\text { Directed } \\
\text { Diffusion }\end{array}$ & $\begin{array}{c}\text { CDMA/ } \\
\text { TDMA }\end{array}$ & Yes & $100 \mathrm{Mbps}$ & Yes & Locally & Moderate & Good \\
\hline
\end{tabular}

\section{CONCLUSION}

Finally by concluding the discussion and coming to the point that due to the vast number applications of WSN's in every field of life, there should be some sort of an algorithm which not only solves the energy issue but also capable of communicating efficiently and rapidly with its servers and nearest processing centers. So that information is routed and received correctly along with the reduction of energy consumption.

S-MAC protocol is the more efficient, more energy conservative, robust, more faster in communication, data loss is minimum, having good scalability, traffic adaptive characteristic and locally synchronized and the most advanced routing protocol explicitly designed for wireless sensor networks.

\section{ACKNOWLEDGMENT}

The success of this research paper would not have been achieved without the unwavering support received from Engineer Umar Mujahid for his continued support and encouragement. I'll remain forever indebted to him. This was indeed a worthy exercise, and I hope that the outcome can be used as reference for the future implementation of this technology in Pakistan.

Last but not the least, I place a deep sense of gratitude to my loving parents whose words of encouragement and a push for tenacity still ring in my ears. Without their support and indefatigable faith in me, none of my achievements would have been possible. I would also like to acknowledge my fiancée who has been a constant source of inspiration during the preparation of this work. 


\section{REFERENCES}

[1] Ian F. Akyildiz, Weilian Su, Yogesh Sankarasubramaniam, and Erdal Cayirci, "A Survey on Sensor Networks", Communications Magazine, IEEE (Volume: 40, Issue: 8), August 2002.

[2] Ilker Demirkol, Cem Ersoy, and Fatih Alagöz, Bogazici University, MAC Protocols for Wireless Sensor Networks: A Survey”, IEEE Communications Magazine, April 2006.

[3] Chalermek Intanagonwiwat, Ramesh Govindan, et al,“ Directed Diffusion for Wireless Sensor Networking", IEEE/ACM TRANSACTIONS ON NETWORKING, VOL. 11, NO. 1, FEBRUARY 2003.

[4] Macros Augusto M.Vieira, Diogenes Cecilio da Silva Junio, "Survey on Wireless Sensor Network Devices", IEEE, 2003.

[5] Th. Arampatzis, J. Lygeros and S. Manesis, "A Survey of Applications of Wireless Sensors and Wireless Sensor
Networks", Proceedings of the $13^{\text {th }}$ Mediterranean Conference on Control and Automation Limassol, Cyprus, June 27-29, 2005.

[6] IAN F. AKYILDIZ, "A Survey on Wireless Mesh Networks", IEEE Radio Communications, September 2005.

[7] Seema Bandyopadhyay and Edward J. Coyle, Energy Efficient Hierarchical Clustering Algorithm for Wireless Sensor Networks", IEEE, 2003.

[8] Wei Ye, John Heidemann, Deborah Estrin, "An EnergyEfficient MAC protocol for wireless sensor networks", IEEE INFOCOM, 2002.

[9] Swati Sharma, Dr. Pradeep Mittal, "Wireless Sensor Networks: Architecture, Protocols", International journal of advanced research in computer science and software engineering, Vol. 3, Issue 1, January 2013. 